2004 Command and Control Research and Technology Symposium The Power of Information Age Concepts and Technologies

\title{
APPLICATION QOS BASED TIME-CRITICAL MACHINE-TO-MACHINE RESOURCE MANAGEMENT IN BM/C2 SYSTEMS
}

\author{
E. Douglas Jensen \\ The MITRE Corporation \\ 202 Burlington Road, Bedford, MA 01730 \\ Voice 781-271-2514, Fax 781-271-4686 \\ jensen@[mitre,real-time].org \\ http://www.real-time.org
}




\title{
Application QoS Based Time-Critical Machine-to-Machine Resource Management in $B M / \mathrm{C}^{2}$ Systems
}

\author{
E. Douglas Jensen \\ The MITRE Corporation \\ Bedford, MA 01730 \\ jensen@[mitre,real-time].org
}

\begin{abstract}
This paper summarizes a novel paradigm for expressing, enforcing, and formally reasoning about time-criticality of machine-to-machine resource management in battle management (BM) and $C^{2}$ systems. Such systems are largely dynamic and asynchronous, and have time frames of $\mathrm{O}\left(10^{-1}\right)$ seconds and higher. Thus they fall into a neglected gap between traditional static periodic "real-time" systems, and traditional "any time" scheduling/planning systems (e.g., for logistics). The paradigm uses application-level QoS metrics (such as track quality, circular error probable, etc.) to derive utility functions for completing tasks, and then uses those utility functions for resource management. This paradigm has been successfully employed in several experimental $B M / C^{2}$ demonstration systems, and is the topic of on-going research by industry and academia.
\end{abstract}

\section{Keywords}

Time-critical, real-time, resource management, scheduling, QoS, utility, time/utility functions, utility accrual

\section{Introduction}

A system provides services, each of which may have various qualities. Each service usually has multiple dimensions of quality. Generic examples include timeliness, availability, and security. Quality of service (QoS) is traditionally associated only with network communications e.g., bandwidth, BER, latency and jitter. But QoS is an inherently general concept that can be employed at any levels of a system and enterprise.

In many systems, complex dynamic service and thus resource - conflicts and dependencies arise. Resolution of the resource contention affects the quality of the provided services to the users and thus the success of the mission and the enterprise. Not all service requests can always be perfectly satisfied; adaptive situation and application-specific resource management decisions are required.

Some of the service requests are time-critical - the quality of the service depends on when it is performed. The timeliness of these services may have potentially drastic impacts on the enterprise and its mission, including survival of property and human lives.

By popular convention, the term "safety critical" is reserved for very simple, static, systems that can be developed in accordance with DO178B and other similar standards. But many large scale, dynamic, systems (such as for combat platform mission management, and battle management) have at least as much potential for human harm as do those "safety critical" ones, yet they are beyond the foreseeable state of the art in high assurance in the spirit of DO-178B et al. In the absence of substantive research results on that problem, assurance must be sought by design methodologies, simulations, and extensive testing.

In this paper we discuss resolving resource contention by adaptively maximizing total $a p-$ plication-level quality of service ("AQoS") of a set of services according to application- and situation-specific criteria. Multimedia and similar applications popular in the real-time research community have AQoS metrics, but they are isomorphic to the QoS metrics of traditional networking, and so do not present significantly new resource management challenges and op- 
portunities. We are concerned with managing application, as well as system, resources (hardware and software) - above, as well as at, the network level - using AQoS metrics, such as (in most systems of interest to us) track quality and weapon spherical error probable.

Application designers often think in terms of what we refer to as AQoS metrics, but not in a general and methodological way. Instead, they consider certain metrics and use their domain expertise to attempt to aggregate these into the proper "tuning" of the system. System and resource management (OS, middleware) software designers usually do not think in terms of AQoS. Thus, there are needs for systematizing and quantifying: the expression of AQoS metrics, and the potential trade-offs among them, to resource management software; and managing resources adaptively by employing AQoS to dynamically optimize the system's behavior according to the users' wishes.

We are particularly interested in helping meet those needs in the context of satisfying application timeliness requirements. We are focused on using AQoS metrics in defining how each service's timeliness contributes to its utility to the current state of the mission and sequencing access to shared resources based in part on maximizing the utility accrued from the set of services.

\section{Dynamic Time-Critical Control Systems}

Many important time-critical control systems do not fit the "real-time" stereotype of: being small scale, static, periodic, centralized; performing autonomic monitoring and control of simple devices; and having time frames in the microsecond and millisecond range. Instead, they: are large scale in various dimensions (e.g., millions of lines of source code), dynamic, "mesosynchronous," and distributed; perform closed loop automated control at any level(s) of an enterprise; and typically operate in time frames of hundreds of milliseconds to several minutes.

In mesodynamics [1], the prefix meso refers to the middle ground between classical physics and quantum physics. By mesosynchronous real-time systems we mean those that are in the middle ground between totally synchronous - in the sense of having only static, periodic, timedriven, activities (e.g., [2]) and totally asynchronous - in the sense of having only dynamic, aperiodic, event-driven, activities. The real world has many important real-time control systems at various points in this mesosynchronous middle ground. The derivation of "mesosynchronous" from "mesodynamics" also reflects that synchronous real-time computing, like classical physics, is relatively well understood, while asynchronous real-time computing, like quantum mechanics, requires a paradigm shift and thus a great deal more research and development on methodologies and formalisms.

It might be tempting to erroneously interpret "mesosynchronous" as meaning that a system is composed of separate traditional synchronous static hard real-time, and asynchronous dynamic, non-real-time parts. While mesosynchronous systems normally do have traditional synchronous hard real-time parts, the asynchronous parts are just as "real-time" as the synchronous ones are. And some parts are neither synchronous nor asynchronous - or are both. Properly speaking, a real-time activity is one that has a completion time constraint. Asynchronous activities may have deadlines, even hard deadlines that if missed result in operational failures - assurances about their timeliness are based on adherence to resource management policies, and are almost always unavoidably non-deterministic. More commonly, these activities have softer but more complex time constraints, and sequencing optimality criteria that are softer but more complex than simply always meeting all deadlines (e.g., minimize the expected completion time tardiness accord- 
ing to activity importance). That does not mean these activities are in any way less "important" or less mission-critical or even less safetycritical than the synchronous activities - indeed, quite the contrary.

The application domain of primary interest to us is defense. Defense systems and applications have always had by far the most challenging time-critical resource management problems and are MITRE's predominant focus. Examples include: multi-role and multi-mode sensors (e.g., phased array radars such as MP-RTIP [3]), combat and surveillance platform management (e.g., the new E-10A (MC2A) aircraft [4], UAV's and UCAV's [5], the $\mathrm{DD}(\mathrm{X})$ class of destroyers [6], the Army Future Combat System vehicle cluster [7], battle management and mission management, (e.g., time-critical targeting [8]), command and control (e.g., AWACS [9]), and network-centric warfare [10].

Time-critical resource management in network-centric warfare is exemplified by the Affordable Moving Surface Target Engagement (AMSTE) system [11], a notional non-classified depiction of which is in Figure 1. AMSTE includes netted airborne and spaceborne Ground Moving Target Indication (GMTI) sensors for tracking a target on the ground, a missile launching fighter aircraft, and a missile guidance aircraft (which is usually one of the sensor aircraft). The targets are moving or alternately moving and stopping. The AMSTE objectives are: to maintain target track from nomination through engagement, from tactically significant standoff ranges; and to provide precision fire control updates to missiles in flight. These objectives require time-critical orchestration of multiple sensors, data links, and the missile. The control loop has about a one second time constraint for sensor to tracker to missile, and about an eight $\mathrm{Hz}$ update rate to the missile (the details of an actual AMSTE system are classified).

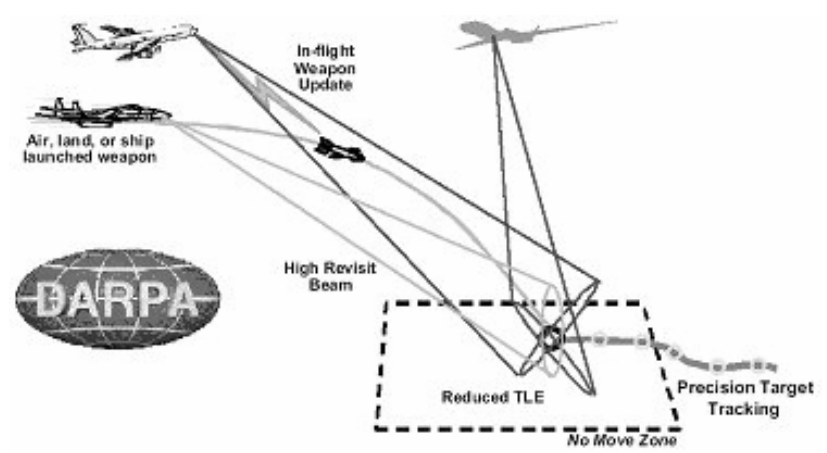

Figure 1. Notional depiction of an AMSTE system

The milliseconds-to-minutes time scale is in a "no man's land" of time-critical resource management theory and practice, between conventional real-time scheduling, and classical logistics (e.g., shop) scheduling with deadlines. Conventional real-time scheduling concepts and techniques: are for static (deterministic), periodic, and (to a degree) sporadic activities, and cannot handle arbitrary asynchrony and dynamic changes in load and resources; timeframes are presumed to be in the microseconds to milliseconds range, which limits resource management algorithm capability. Classical logistics scheduling concepts and techniques [12] include: stochastic as well as deterministic models; but time-frames are in the minutes to hours (usually too computationally intensive for on-line automated resource management); and the use of lateness (deadline - completion time) as the timeliness metric, the linearity of which severely limits time constraint expressiveness.

\section{Time/Utility Functions and Utility Accrual}

In 1977 Jensen created a novel scheduling paradigm for dynamic time-critical systems [13] for the U.S. Army Safeguard Command's AN/FPQ-16 Perimeter Acquisition Radar Characterization System [14], located at what is now the USAF Space Command's Cavalier Air Force Station, in Cavalier, North Dakota. The system's radar performance was not up to expectations. Simulated performance with the new scheduling paradigm was sufficiently improved 
that the paradigm was deemed to possibly violate SALT II, and hence was not deployed. From then until now, Jensen and his collaborators have continued to develop, refine, and apply the paradigm. At Carnegie Mellon's Computer Science Department, two of Jensen's $\mathrm{Ph}$.D. students wrote their theses on this topic $[15,16]$ - devising new scheduling algorithms, proving theorems about them, simulating them, and implementing them in our Alpha real-time distributed OS kernel [17]. Subsequent enhancements have also been made by other researchers (e.g., [18, 19, 20, 21]).

The two keystone concepts in our paradigm are time/utility functions (originally called time/value and then benefit, functions) and utility accrual sequencing (scheduling, dispatching) optimality criteria $[13,22]$.

A time/utility function (TUF) expresses the utility to the system (derived from AQoS metrics) of completing an activity (e.g., service) as an application- or situation-specific function of when it completes. Deadlines are a simple special case, where full utility is achieved if the activity completes by its deadline, and zero (or some negative) utility is achieved if it completes after its deadline. Four example TUF's are depicted in Figure 2.

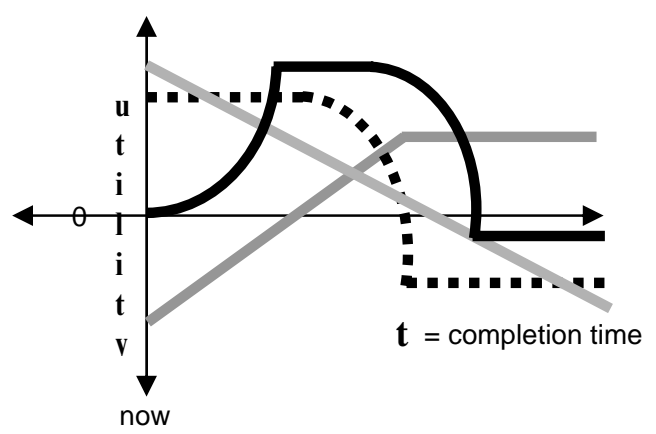

Figure 2. Four example time/utility functions

Utility accrual (UA) based sequencing algorithms sequence activities according to optimality criteria based on accruing utility - such as maximizing the sum of the utilities (plus satisfying dependencies such as precedence, resource constraints, etc.). The algorithms take into account the known or expected durations of the activities, and may be either deterministic or non-deterministic (e.g., stochastic).

\section{Worked Examples}

To illustrate the use of this paradigm, we summarize two significant (unclassified) demonstration applications we implemented successfully in collaboration with application domain experts.

\section{AWACS Surveillance Tracker}

The first application is an AWACS surveillance tracker [23], implemented jointly by the MITRE Corporation and the Open Group. AWACS is an airborne radar system with varied missions, including air surveillance. Surveillance missions generate aircraft tracks for battle management and command and control. It is very common for there to be too many radar reports for the computing system to process, which causes sectors of the sky to "go blank". Higher performance computing only helps somewhat, because the potential report load and the need for ever-better tracking algorithms will always exceed all the computing capacity. Currently, operators have knowledge-intensive manual work-arounds for certain overload situations.

The surveillance mission includes a number of activities; for brevity here, we consider only the most computationally demanding activity, association, which associates radar reports with tracks. There is a multiplicity of concurrent association threads, one for each current radar report.

There are two sensors (radar and IFF) sweeping $180^{\circ}$ out of phase with a 10 -second period, which suggests the association TUF has a "critical time" at the 10-second period length, at least two distinct non-zero utilities before the critical time, and a third distinct, lower, utility after the critical time. 
Prior to the critical time, processing a sensor report for one of these tracks in under five seconds (half the sweep period) would provide better data for the corresponding report from the out-of-phase sensor. So the utility decreases with time. The TUF had to decrease linearly due to an implementation artifact in this experimental system - the OS (OSF/RI's MK7 [24]) TUF scheduling algorithm allowed only one critical time. The slope, and thus $\mathrm{U}_{2}$, were derived empirically (systematizing this process is one of our current research topics).

After the critical time, utility is zero, because newer sensor data has probably arrived if the processing load in one sensor sweep period is so heavy that it couldn't be completed, probably the load will be about same in next period, so there will be no capacity to also process data from the previous sweep. A tracker that could process older as well as current data would be significantly more complex and probably delay the track update. The resulting TUF shape is shown in Figure 3.

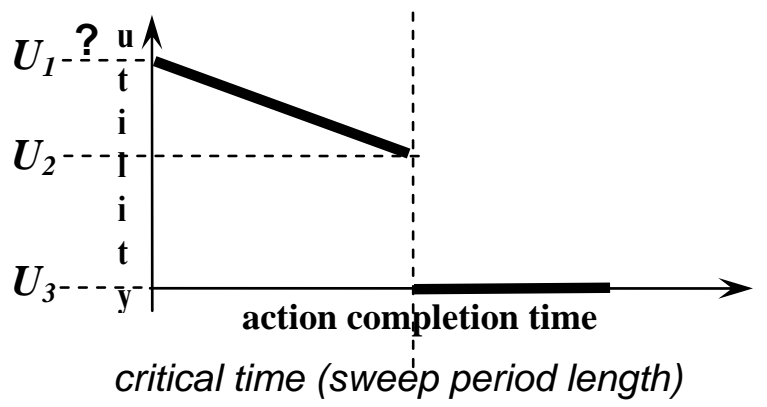

\section{Figure 3. Association time/utility function shape}

Next, the utility value $U_{1}$ had to be determined. To do that, we used well-established surveillance tracker AQoS metrics, which are based on the tracker domain experts' knowledge about how to manually attempt to resolve contention for resources:

- Don't drop tracks, because they are expensive to re-create
- User-identified "important" tracks receive preference

- User-identified "important" geographic regions receive preference

- Maneuvering tracks need to be updated more frequently than non-maneuvering tracks

- Potentially high threat tracks receive preference

- High speed tracks receive preference

- Tracks with poor state estimates receive preference.

Conventionally, tracker domain experts aren't provided with computation technology that would give them incentives to use their knowledge to understand and express behavioral options and trade-offs in the face of dynamic uncertainties (i.e., gracefully handling overloads) that plague current trackers. Our intention was to provide technology that would utilize tracking domain expertise sufficient to automate the adaptive assignment of the right resources to the right tasks at the right time.

We chose three (to limit the complexity of the experimental system) AQoS metrics for an AWACS surveillance tracking application (a decision with which the domain experts concurred):

- Quality - 0 to 7: a traditional measure of the amount of recent sensor data incorporated in a track record, and incremented or decremented after each radar scan

- Accuracy - "high" or "low": a measure of the uncertainty of the estimate of a track's position and velocity and derived from Kalman filter processing

- Importance - "high" or "low": traditionally, operator-identified based on geography, threat, and other characteristics. 
The twelve combinations of these three AQoS metrics are diagrammed in Figure 4.

The initial utility $U_{1}$ of an association for a track report is derived from track AQoS metrics by gedanken experiments. The utilities range from a low of 10 to a high of 6000 - meaning that 600 times more utility is gained by performing an association for a low accuracy, high importance, poor quality track, than for a high accuracy, low importance, high quality track.

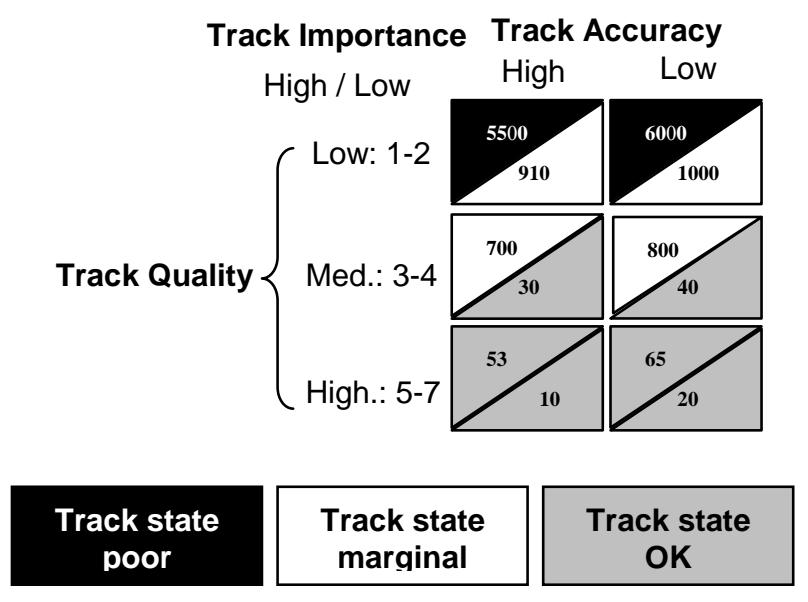

\section{Figure 4. The 12 Combinations of track AQoS's and their relative utilities}

All the association (and other) threads are scheduled based on their utility functions. For the association threads, the tracking application selects the established TUF from the scheduler's library of shapes. The tracking application does a look-up in the utility $\mathrm{U}_{1}$ table for each association thread before calling the scheduler. A utility accrual based processor scheduling discipline (in the OS, in this system) schedules threads according to a heuristic that attempts to maximize total accrued (in this case, summed) utility.

This surveillance tracking application was implemented on a research OS (the Open Software Foundation's MK7) having a scheduling framework that allowed different disciplines to be used. Figure 5 shows two critical tracker AQoS metrics, the number of dropped tracks and track quality, for more and less important threads, using three scheduling disciplines: FIFO (the discipline used in the currently deployed AWACS tracker); priority (the predominant discipline in real-time computing systems); and one of our utility accrual disciplines.

In the measured cases shown, under nonoverload conditions, the tracker handled all tracks as expected and delivered high AQoS in both metrics for all tracks. In an overload scenario, such as when the tracker can only process about $33 \%$ of the input, the system delivered essentially perfect track quality for the more important tracks, while delivering a reasonable track quality for the less important tracks. (In some respects, this overload level can be likened to a system where the probability of detecting an airborne object is about $33 \%$.) When the tracker was further constrained so that it could only process about $10 \%$ of the input, the system finally dropped some tracks - from the less important track class. No important tracks have been dropped during our demonstrations. In addition, the demonstrations have shown that the tracker also adapts when new resources are added. In that case, which we demonstrate by loosening the time constraints on association processing, the prototype automatically delivers approximately the maximum achievable AQoS.

Note that these results are not easily obtainable with static priority tracking systems. In priority-based trackers, track priorities might reasonably be set according to track importance, where high importance implies high priority. In our tracker, scheduling decisions are based on both importance and timeliness, and even relatively unimportant tracks can have very high application utility in a surveillance mission - an outcome that would not be possible with straightforward priority-based scheduling. 


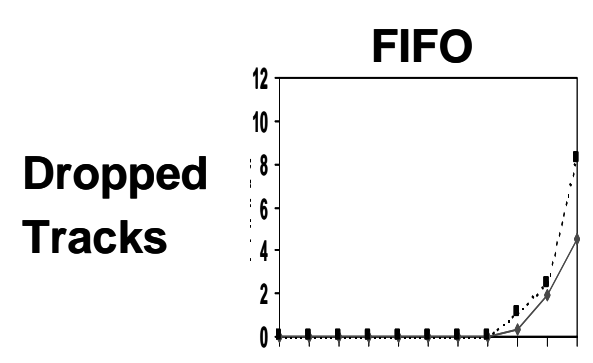

$>1110 \begin{array}{llllllllll}10 & 8 & 7 & 6 & 5 & 4 & 3 & 2 & 1\end{array}$

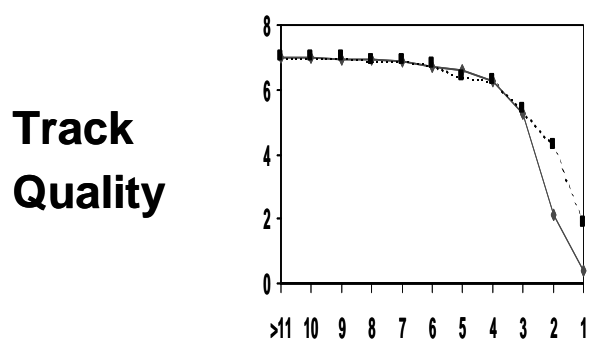

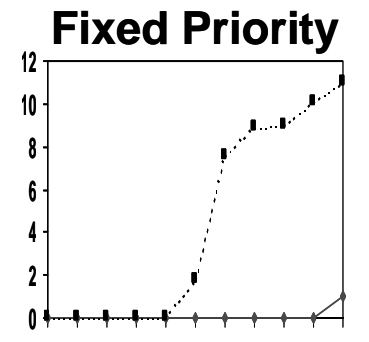

$\begin{array}{lllllllllll}111 & 10 & 9 & 8 & 7 & 6 & 5 & 4 & 3 & 2 & 1\end{array}$

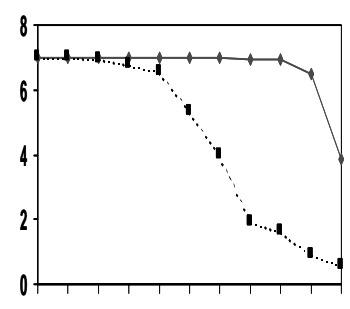

$\begin{array}{lllllllllll}11 & 10 & 9 & 8 & 7 & 6 & 5 & 4 & 3 & 2 & 1\end{array}$

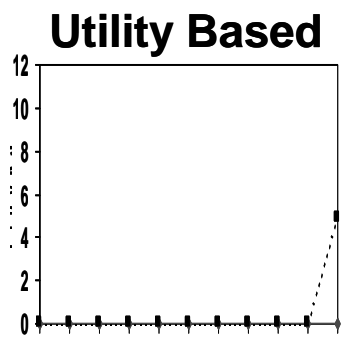

$>1110 \begin{array}{lllllllll}10 & 8 & 7 & 6 & 5 & 4 & 3 & 2 & 1\end{array}$

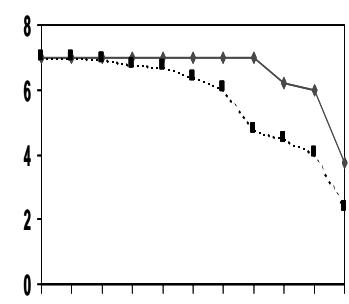

$\begin{array}{lllllllllll}11 & 10 & 9 & 8 & 7 & 6 & 5 & 4 & 3 & 2 & 1\end{array}$

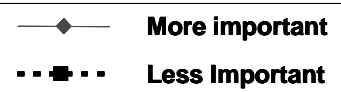

Figure 5. Comparison of AWACS surveillance tracker AQoS metrics for different scheduling disciplines

\section{Battle Management for Coastal Air Defense}

The second application is a notional battle management system for coastal defense from cruise missiles and bombers [25]. It was implemented collaboratively by the General Dynamics Corporation and Jensen's Archons Project at Carnegie Mellon University's Computer Science Department. It is included here to further illustrate the dynamic adaptivity possible with time/utility functions, which would be difficult to achieve with priorities or even deadlines.

This system's mission is to destroy incoming hostile cruise missiles (for brevity here we will disregard the hostile bombers), by using guided interceptor missiles. There may be more cruise missiles and decoys than can be intercepted, due to the number of cruise missiles, insufficient interceptors, and insufficient computational resources for battle management. Cruise missiles maneuver during flight, but do not try to evade the interceptors. Interceptors are guided by airborne defenders using airborne (AWACS, etc.), spaceborne, and ground based, sensor platform data.

The cruise missile defense (CMD) application quality of service (AQoS) metric here is the weapon spherical error probable (WSEP). Spherical error probable (SEP) is the radius in meters of a sphere centered on a point (e.g., the cruise missile) within which the true value of an estimated point (e.g., the interceptor missile) will lie with a probability of 0.5 . The WSEP is an SEP radius around the cruise missile chosen such that if the interceptor gets within that distance, the interceptor's terminal guidance system can take over and with $\rho=0.5$ cause the interceptor to either impact the cruise missile or detonate close enough to the cruise missile to destroy it.

Some CMD AQoS optimality objectives are:

- minimizing WSEP

- intercepting sooner (further from the cruise missile's target) is better (EMP, etc.) 
- intercepting before the cruise missile reaches its target is better

- intercepting away from an adverse geographical location (e.g., over a populated area) is better

- intercepting before the blast damage to the cruise missile's target from the combined cruise missile and interceptor missile would exceed the blast damage from the cruise missile explosion alone is better.

WSEP is affected by several factors, including:

- guidance updates to the interceptor are repetitive but not necessarily periodic or even sporadic, and need to occur more often as the interceptor gets closer to the cruise missile

- it becomes more important to use the most recent information about the positions and velocities of the missiles as the distance between the cruise missile and the interceptor decreases

- $\quad$ some cruise missile targets, and thus cruise missiles, are more important than others.

The CMD application consists of a number of activities, as depicted in Figure 6.

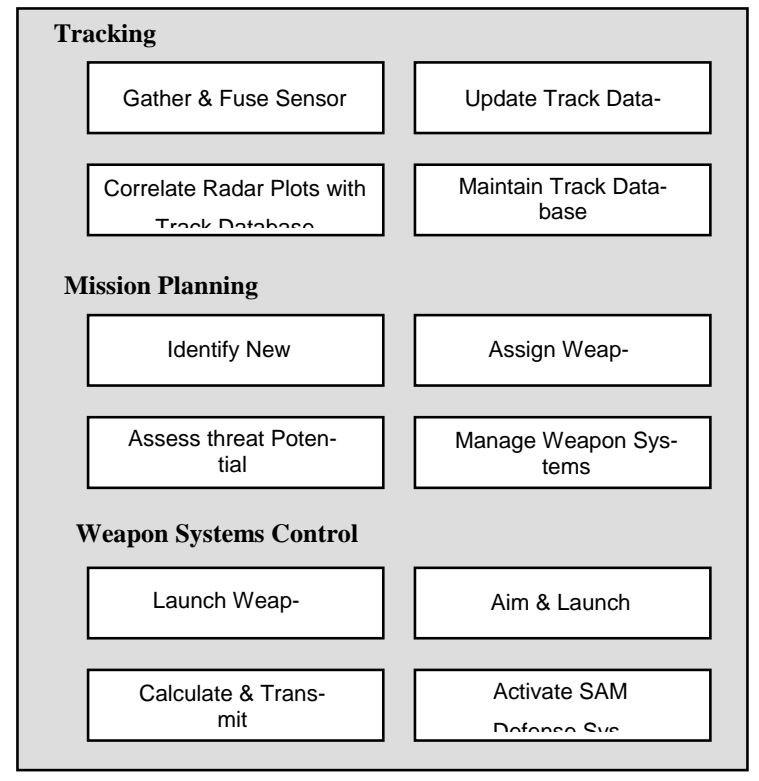

\section{Figure 6. The coastal air defense battle management activities}

Some activities resemble corresponding activities in the AWACS surveillance tracker. The plot correlation and track database maintenance threads have critical times corresponding to the radar frame arrival rate. In both cases, it is better if the processing is completed before the next frame of sensor data arrives. It is acceptable for the processing to slip as much as one additional time frame under extreme overload situations. The plot correlation activity has a much greater utility to the system under overload conditions. The TUF's for those two threads are shown in Figures $7 \mathrm{a}$ and $7 \mathrm{~b}$.
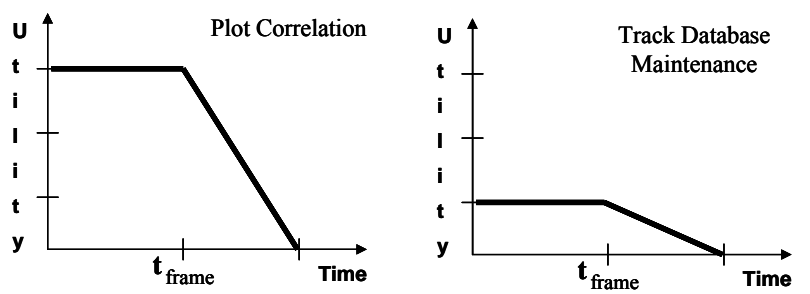

Figures 7 a,b. The plot correlation and track database time/utility functions

But the timeliness requirements for the interceptor missile control threads are more complex because they vary over the course of an interception engagement. After an interceptor is launched, the guidance control threads must issue timely aperiodic course updates to ensure a successful intercept. The required timeliness of these updates, and the importance of completing the course corrections at the desired time, change as the distance decreases between the interceptor and the cruise missile, and between the cruise missile and the coastline. Each interceptor's control thread changes three parameters of its shape during the engagement, as shown in Figure 8. Figure 9 shows three representative snapshots of this adaptivity as it progresses from right (launch) to left (intercept). 


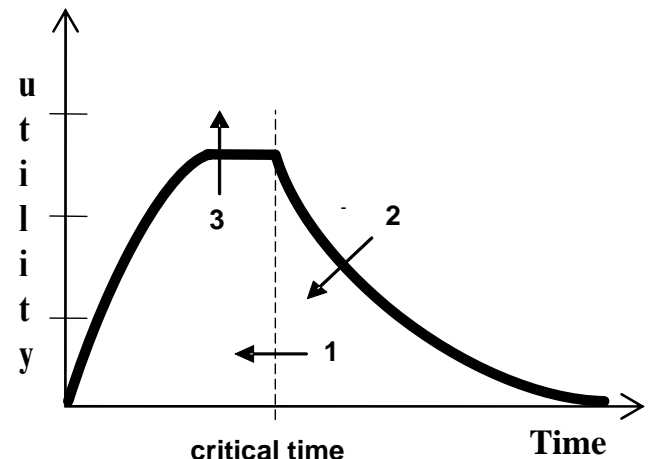

Figure 8. The interceptor time/utility function has three shape parameters that adapt during the engagement

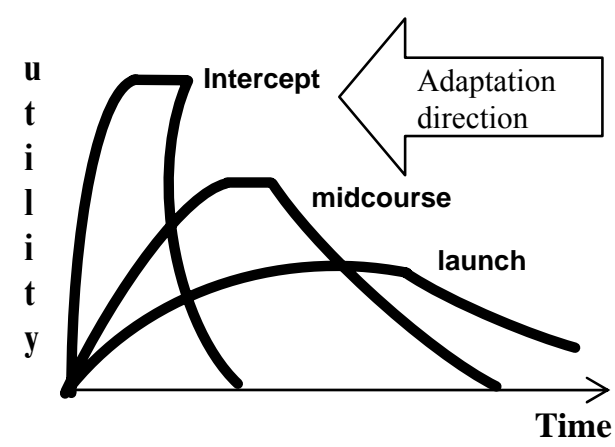

Figure 9. Three representative snapshots of the interceptor time/utility function adapting during the engagement

This effect is extremely difficult to achieve by manipulating priorities or deadlines.

\section{Conclusion}

Our current research objective on this topic is to advance this paradigm's concepts and techniques, and to explore its applications in the $\mathrm{BM} / \mathrm{C}^{2}$ domain. We are devising new resource management algorithms, proving timeliness and other properties, doing simulations of them, building and measuring experimental implementations of them, devising a methodology for users to apply the paradigm, and constructing a proof of concept software tool to facilitate the methodology.

\section{References}

[1] R.D. Peters, http://mathforum.org/library/view/16558.html

[2] H. Kopetz, Real-Time Systems, Design Principles for Distributed Embedded Applications, Chapter 8, "The Time-Triggered Protocols," Kluwer Academic Publishers, 1997.

[3]

http://www.globalsecurity.org/intell/systems/mprtip.htm

[4] http://esc.hanscom.af.mil/esc-mc2a/

[5]

http://www.globalaircraft.org/planes/?type=uav

[6] http://peoships.crane.navy.mil/ddx/

[7] http://www.darpa.mil/fcs/

[8]

http://www.globalsecurity.org/space/systems/bm c3i.htm

[9]

http://www.globalsecurity.org/military/systems/ai rcraft/e-3.htm

[10]

http://www.dodccrp.org/ncwPages/ncwPage.html [11]

http://www.globalsecurity.org/military/systems/ munitions/amste.htm

[12] M. Pinedo, Scheduling: Theory, Algorithms, and Systems, 2nd edition, Prentice Hall, 2002, ISBN 0-130-28138-7.

[13] M. G. Gouda, Y-W. Han, E. D. Jensen, W. D. Johnson, and R.Y. Kain, Distributed Data Processing Technology, Vol. IV, Applications of DDP Technology to BMD: Architectures and Algorithms, Honeywell Systems and Research Center, Minneapolis, MN. September 1977.

\section{[14] http://srmsc.org/}

[15] C. D. Locke, Best-Effort Decision Making for Real-Time Scheduling,, Ph.D. Thesis, CMUCS-86-134, Department of Computer Science, Carnegie Mellon University, 1986. 
[16] R. K. Clark, Scheduling Dependent RealTime Activites, Ph.D. Thesis, CMUCS-90-155, School of Computer Science, Carnegie Mellon University, 1990.

[17] R. K. Clark, E. D. Jensen and F. D. Reynolds, "An Architectural Overview of the Alpha Real-Time Distributed Kernel", USENIX Workshop on Microkernels and other Kernel Architectures, pp 200-208, 1993.

[18] P. Li, B. Ravindran, H. Wu, and E. D. Jensen, A utility accrual scheduling algorithm for real-time activities with mutual exclusion resource constraints, IEEE Transactions on Computers. Submitted August 2003.

[19] D. Mosse, M. E. Pollack, and Y. Ronen, "Value-density algorithm to handle transient overloads in scheduling," in Proc. Euromicro Conference on Real-Time Systems, June 1999, pp. 278-286.

[20] W. T. Strayer, "Function-driven scheduling: A general framework for expressing and analysis of scheduling," Ph.D. dissertation, University of Virginia, May 1992, department of Computer Science.

[21] S. A. Aldarmi and A. Burns, "Dynamic value-density for scheduling real-time systems," in Proc. of Euromicro Conference on Real-Time Systems, June 1999, pp. 270-277.

[22] E. D. Jensen, C. D. Locke, and H. Tokuda, "A Time-Driven Scheduling Model for RealTime Systems," Proc. Real-Time Systems Conference, IEEE, December 1985.

[23] R. Clark, E. D. Jensen, A. Kanevsky, J. Maurer, P. Wallace, T. Wheeler, Y. Zhang, D. Wells, T. Lawrence, and P. Hurley, "An adaptive, distributed airborne tracking system," in Proceedings of IEEE International Workshop on Parallel and Distributed Real-Time Systems, ser. Lecture Notes in Computer Science, vol. 1586. Springer-Verlag, April 1999, pp. 353-362.

[24] D. Wells, "A Trusted, Scalable, Real-Time Operating System," Dual-Use Technologies and Applications Conference Proceedings, pp II 262270, Utica, NY, 1994.

[25] D. P. Maynard, S. E. Shipman, and R. K. Clark. et al., "An example real-time command, control, and battle management application for alpha," Technical Report Archons Project TR88121, CMU Computer Science Department, December 1988 . 